\title{
Modelo de gestión de redes de distribución mediante UML y BPL
}

\section{Management model of distribution networks through UML and BPL}

Luis Alejandro Arias Barragán

Ingeniero Electromecánico, Magíster en Ciencias de la Información y las Comunicaciones. Docente de la Universidad Autónoma de Colombia. Bogotá, Colombia.

Contacto: lincarias@yahoo.com

Diego Fernando García

Ingeniero Eléctrico, Magíster en Sistemas de Generación, Doctor en Ingeniería Eléctrica, Electrónica y Automática. Docente de la Universidad del Valle. Cali, Colombia.

Contacto: diego.garcia@correounivalle.edu.co

\section{Edwin Rivas Trujillo}

Ingeniero Eléctrico, Magíster en Sistemas de Generación, Doctor en Ingeniería Eléctrica, Electrónica y Automática. Docente de la Universidad Distrital Francisco José de Caldas. Bogotá, Colombia. Contacto: erivas@udistrital.edu.co

Fecha de recepción: 12 de diciembre de 2012

Fecha de aceptación: 21 de mayo de 2013
Clasificación del artículo: Investigación

Financiamiento: Universidad Autónoma de ColombiaUniversidad Distrital Francisco José de Caldas

Palabras clave: BPL, despacho, GD, red de distribución, UML.

Key words: BPL, dispatch, GD, distribution network, UML.

\section{RESUMEN}

En este artículose propone un modelo de gestión del proceso de despacho en sistemas alimentados por generación eléctrica convencional y distribuida, implementando para ello un sistema automatizado que permita de manera dinámica adaptarse a escenarios de cargas variables, aleatoriedad de los parámetros de eficiencia y calidad de energía del sistema, restricciones técnicas, económicas y regulatorias, y la inclusión activa de agentes generadores distribuidos.

\begin{abstract}
This paper proposes a model of the clearance process management in systems powered by conventional electric and distributed generation, there by implementing a system which allow to dynamically adapt to scenarios of varying loads, randomness of the parameters of efficiency and power quality of the system technical constraints, economic and regulatory, as well as the active participation of agents distributed generators.
\end{abstract}




\section{investigación |}

\section{INTRODUCCIÓN}

El surgimiento y masificación de las fuentes renovables de energía (solar, eólica, geotérmica, etc.) ponen de manifiesto la necesidad de buscar nuevos esquemas de regulación, de control del proceso de despacho energético y en general de la gestión, que permitan incluir las nuevas condiciones de mercado y las implicaciones técnicas y tecnológicas que se presentan con los nuevos actores (clientes activos consumidores-generadores, clientes consumidores con poder de decisión sobre su demanda y cargas altamente aleatorias como los vehículos eléctricos). Por ende el comportamiento dinámico de los sistemas de distribución hace necesario involucrar un modelo de gestión que brinde soluciones versátiles y adaptables a escenarios diversos de demanda, donde primen los criterios de eficiencia y calidad de energía.

En este artículo se realiza en primera instancia una descripción de los tipos de despacho energético con fuentes convencionales y con generación distribuida. Posteriormente se plantea el modelo de gestión de manera general, dándose paso al detalle de las diferentes operaciones y escenarios que enfrentará la gestión de los sistemas, mediante casos de uso en el lenguaje de modelamiento Unified Modelling Language (UML). Finalmente, se realizan pruebas de conectividad con tecnología BPL en escenarios industriales como caso de estudio típico en redes de distribución.

\section{DESPACHO ENERGÉTICO EN REDES DE DISTRIBUCIÓN}

\subsection{Despacho energético en redes de distribución con fuentes convencionales de energía}

El despacho es el proceso por el cual se realiza la asignación de cargas a los diferentes agentes proveedores de energía. En este proceso se busca repartir la demanda total del sistema entre los generadores disponibles de forma que el coste global de generación sea el mínimo sujeto a restricciones técnicas, tecnológicas y ambientales.

La planeación del despacho energético tiene como finalidad evitar la desatención de la demanda, prevenir colapsos en el sistema de potencia y disminuir los tiempos de racionamientos y de restablecimientos del servicio, parámetros que constituyen los principales criterios de evaluación de la confiabilidad del sistema de generación, transmisión y distribución eléctrica [1].

El problema de garantizar condiciones factibles de operación en un sistema de despacho energético se resuelve habitualmente sobre la base de un despacho diario. Para este tipo particular de despacho se considera la opción de acoplar o bien desacoplar tanto eslabones individuales como grupos de generación según la variación de la demanda horaria. Sin embargo, cuando son incluidos agentes de generación distribuida surgen inconvenientes ligados a la adecuada integración de las tecnologías y problemas de balanceo de cargas abastecidas con GD [2], [3]

La inclusión de las fuentes de generación distribuida (turbinas eólicas, celdas fotovoltaicas, sistemas biomásicos, pequeñas centrales hidroeléctricas $[\mathrm{PCH}]$, plantas diesel y sistemas FLYWEEL[4]) en el sistema de despacho constituye un factor importante desde el punto de vista de la no-linealidad para todo el proceso de distribución.

\subsection{Despacho con generación distribuida}

Según el Electric Power Research Institute (EPRI), la Generación Distribuida (GD) se interpreta como "la utilización de tecnologías modulares de generación de pequeña capacidad, dispersas a lo largo del sistema de distribución".

La GD ofrece beneficios como la reducción en el flujo de potencia a través de las redes de transporte 


\section{|investigación}

y distribución, alivia el crecimiento de la carga, reduce pérdidas en transmisión y distribución, además de que mejora la calidad del servicio eléctrico y la confiabilidad del sistema de distribución. No obstante, la inserción de GD en sistemas convencionales de distribución acarrea algunos inconvenientes, debido a que estos sistemas fueron diseñados para flujo de potencia unidireccional, es decir, desde las subestaciones hacia los usuarios finales.

Dentro de los inconvenientes cabe resaltar los derivados por fallas temporales, donde la corriente que es inyectada por parte de la GD puede ocasionar averías en los fusibles antes de la primera operación rápida de los re-conectores ubicados "aguas arriba" del sistema de distribución. De la misma manera, se debe anotar que cuando la penetración de GD en un circuito de distribución es grande (por encima del $35 \%$ ), una falla en un circuito aledaño puede ocasionar la apertura del interruptor principal del circuito donde se conecta el eslabón de GD.

En lo referente a las protecciones del sistema de distribución, se debe tener en cuenta que la corriente de carga que suministra la GD puede modificar el alcance de los equipos de protección, haciendo variar la sensibilidad de los instrumentos, ocasionando que se necesite una mayor corriente de falla para causar la reacción de la protección del sistema. De otra parte la no-linealidad que aportan las GD, y la reducción de la confiabilidad en materia de protecciones del sistema requieren una continua adaptación a escenarios dinámicos. En este sentido, se trabaja en la optimización de algoritmos clásicos para el despacho operacional, que incluyen eslabones de control de deslastre, conexión y re-conexión de carga [12]; asimismo se han desarrollado algoritmos adaptativos para la planeación de redes de distribución con inclusión de GD [11], donde se propone la gestión de la red de distribución a través de funciones multiobjetivo, solucionándolas mediante algoritmos genéticos.

Los inconvenientes mencionados ponen de manifiesto un nuevo escenario en el cual el despacho en la red de distribución con generación distribuida deberá introducir nuevos criterios referentes a confiabilidad y seguridad, así como estrategias que involucren agentes activos y pasivos de la red.

A.M. Abdullah [5] plantea un modelo de despacho basado en el análisis de la topología de la red, medición del flujo de potencia antes y después de una avería, mecanismos de deslastre, desconexión y transferencia de carga. En dicho modelo las pérdidas de carga se calculan a través de índices cuantitativos ante diversos grados de contingencia, lo que permite así evaluar la seguridad y vulnerabilidad del sistema.

Diversas estrategias se han contemplado para el control de redes que involucren generación distribuida. A manera de ejemplo, en [6] se propone una metodología para evaluar el punto de equilibrio de las cargas ante perturbaciones en la red, teniendo un elemento de generación distribuida como respaldo. Otra estrategia es ver a los generadores y cargas asociadas como un subsistema denominado micro red o microgrid [7]. Este enfoque permite el control local de la generación distribuida, reduciendo o eliminando la necesidad de una central de despacho.

\section{LENGUAJE DE MODELAMIENTO UNIFICADO (UML)}

El modelamiento de un sistema automatizado desde sus niveles superiores de gestión, utilizando componentes de software, debe poseer como cualidad característica la independencia de cualquier tecnología o lenguaje de programación [13], razón por la cual se escoge UML como sistema de desarrollo de software libre.

UML se define como un lenguaje gráfico para visualizar, especificar, construir y documentar los artefactos de un sistema con gran cantidad de software. Proporciona una forma estándar de diagramar planos de un sistema, abarcando las partes conceptuales (funciones del sistema, y en principio también procesos industriales), y los 
objetos concretos (clases escritas en lenguajes de programación específico, esquemas de bases de datos, componentes de software reutilizables) [14].

UML es un lenguaje de modelado universal, por lo que cada vez más, es empleado para la descripción de arquitecturas. En este caso, se usa para la descripción de la arquitectura de componentes genéricos, $\mathrm{y}$ en particular, para especificar la estructura interna de los elementos arquitectónicos mediante los diagramas de clases y de secuencia [15].

La principal ventaja de UML es que constituye un lenguaje de propósito general, aunque esto en ocasiones puede llegar a convertirse en una desventaja, porque no se pueden representar en toda su dimensión y detalle las situaciones o características propias de dominios específicos [16].

En[17], los autores muestran como a través de UML se puede hacer el modelamiento de las instancias de gestión y supervisión de los procesos en una planta de automatización industrial, coordinando todos los elementos encaminados a dar respuesta a los requerimientos de los clientes.

\section{TECNOLOGÍA BPL/PLC (BROADBAND POWER LINE/POWER LINE COMMUNICATION)}

Los estudios realizados sobre la conveniencia de la transmisión a través de líneas de potencia han cobrado gran importancia en el contexto mundial por cuanto plantean la utilización de un canal de fácil acceso que hasta el momento ha sido subutilizado[20]. El impacto de la utilización de las redes eléctricas como medio de comunicación de datospara países en vías de desarrollo permitiría saltar una enorme brecha en materia de desarrollo con respecto a países vanguardistas y que precisamente está ligada a las limitantes en cobertura de lasredes de datos convencionales. No obstante, existen problemas ligados a la confiabilidad y a la seguridad de dichas redes cuando de ofrecer una buena calidad de servicio (Quality of Service) se trata y que responda a los mínimos lineamientos que formas ya erigidas como convencionales (UTP, fibra óptica, WIMAX, WIFI) ofrecen en la actualidad.

\section{MODELO METODOLÓGICO DE LA PROPUESTA}

En la figura 1 se plantea el modelo metodológico de gestión de manera general, el cual está enfocado a una red de distribución específica dentro del área de influencia de las empresas distribuidoras de la región. El modelo muestra las capas estructurales que tendrá el sistema de gestión desde el punto de vista de su diseño: capa de interfaz gráfica, capa de negocios y capa de base de datos o persistencia,para el proceso de despacho energético eléctrico en escenarios de generación compartida convencional y distribuida, con inclusión de restricciones técnico-tecnológicas y económicas. Para el diseño del modelo del sistema de gestión, en su estructura lógica funcional,se llevará a cabo el desarrollo de herramientas de software que incluirán algoritmos adaptativos (de programación heurística o bien lógica difusa) y el diseño de la infraestructura de dispositivos para el control de los diferentes eslabones funcionales involucrados en el proceso de distribución en la red de despacho objeto del proyecto. De otra parte el diseño del modelo de gestión planteael desarrollo de una red automatizada para la gestión en el proceso de distribución de la energía eléctrica, apoyada en la tecnología BPL. 


\section{investigación}

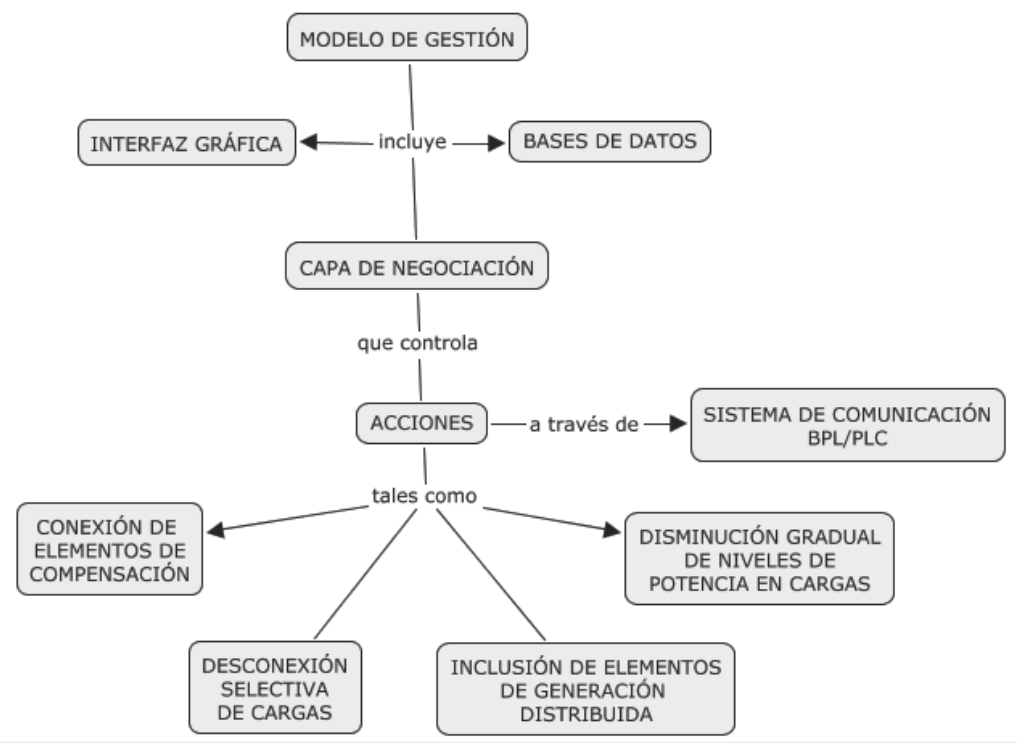

Figura 1. Modelo de gestión para la red de distribución.

Fuente: elaboración propia.

En la figura 2 se muestran detalladamente las diferentes situaciones y escenarios que enfrentará un eventual operador del sistema parala gestión.Las situaciones y escenarios se ilustran a través de los casos de Uso con modelamiento UML. La diagra- mación de los casos de Uso es un insumo importante para la posterior generación de los diagramas de clase y de secuencias lógicas del modelo de gestión que se propone.

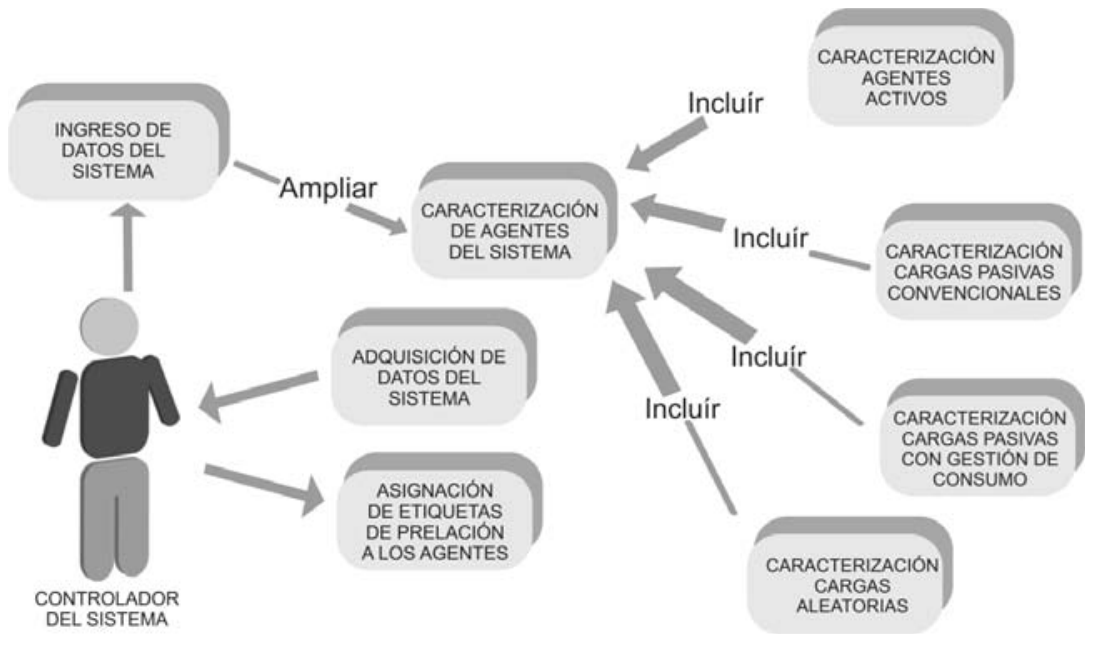

Figura 2. Diagrama de casos de uso para el sistema de gestión de la red de distribución. Fuente: elaboración propia. 


\section{investigación |}

El diagrama de caso de Uso de la figura 2requiereincluir una interfaz gráfica para el sistema de gestión, de acuerdo al modelo de tres capas, cuyas siglas en inglés son GUI, BUSSINES LAYER and
$\mathrm{BD}$, y en español interfaz grafica de usuario, capa de negocio y base de datos. En la figura 3 se plantea la estructura de la interfaz gráfica.

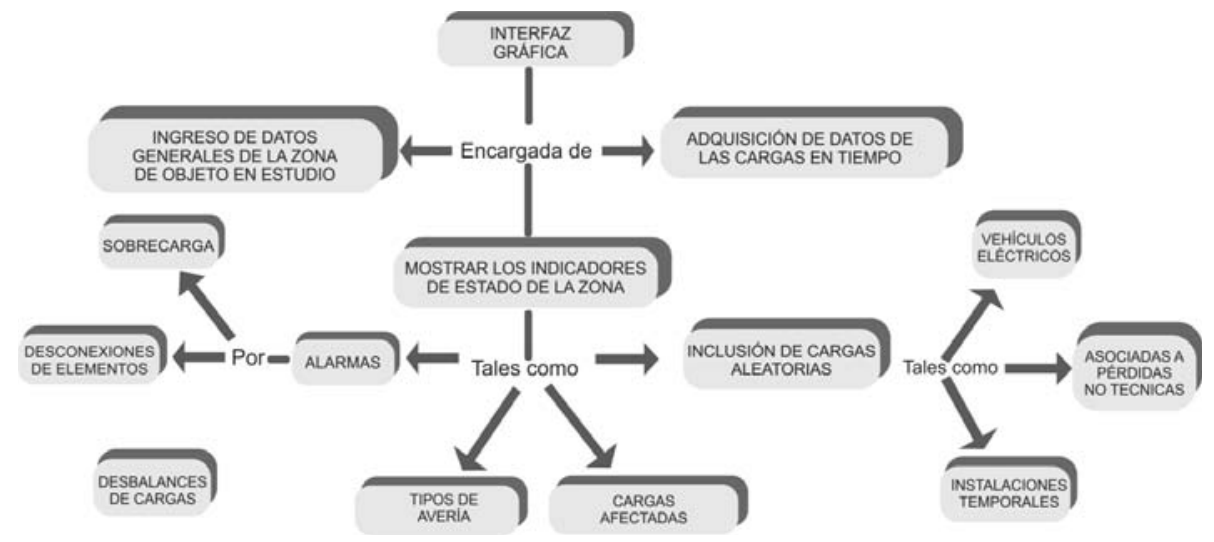

Figura 3. Modelo de interfaz gráfica para la red de distribución.

Fuente: elaboración propia.

La realización del proyecto contempla una serie de etapas que van desde una selección inicial de la zona de distribución que se considere potencialmente más viable para la exploración, hasta la posible implementación de la solución a desarrollar. Para dicha selección se requiere información como población objetivo del proyecto, históricos de demandas y fallas, entre otros datos, atendiendo obviamente los requerimientos que se estimen convenientes de parte de usuarios comoempresas, y en general otras cargas o usuarios característicos del lugar. La estructura lógica de las etapas a seguir se puede ver en la figura 4 .

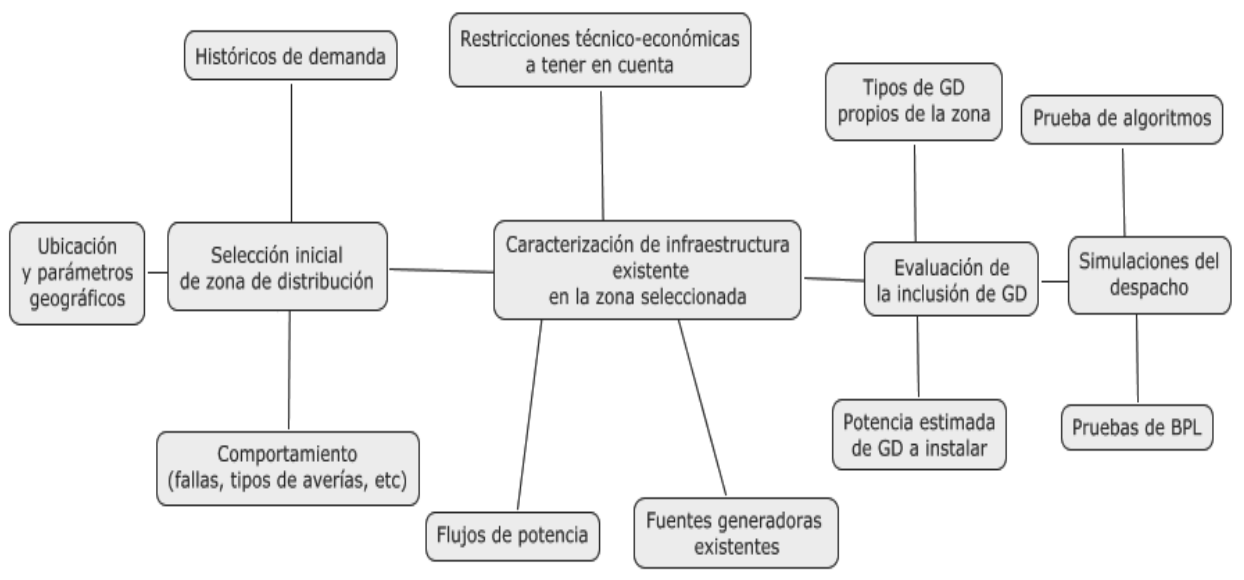

Figura 4. Etapas del proyecto.

Fuente: elaboración propia. 
La etapa siguiente comprende una caracterización plena de los eslabones funcionales involucrados en el proceso de despacho energético de la zona de estudio seleccionada, tales como agentes generadores convencionales, consumidores que se abastecen, niveles de potencia y sus respectivas gráficas de flujos de potencia diaria, semanal, mensual y las restricciones técnicas funcionales con que se cuente en la zona.

Posteriormente se realizará una evaluación de la inclusión de fuentes de generación distribuida presentes en la zona dentro del sistema de distribución existente, y la simulación de escenarios potenciales de despacho, donde se tengan aportes de energía de generadores convencionales como hidroeléctricas y termo-eléctricas junto a fuentes renovables de energía eólica, solar térmica y fotovoltaica,entre otras.

Si bien son una alternativa aceptable de suministro de energía en zonas aisladas de la geografía nacional, o como soporte redundante para consumidores no-regulados, las fuentes renovables de energía se constituyen en "cargas no-lineales" para el sistema de distribución convencional, y se convierten en factores determinantes de un gran número de fallas en la operación normal del sistema eléctrico en general.

Es importante anotar el hecho de que la gran mayoría de las fuentes renovables implementadas en generación distribuida (GD) basan su funcionamiento en procesos de inversión de voltajes DC (corriente directa) a $\mathrm{AC}$ (corriente alterna), convirtiéndose así en fuentes de armónicos en la red que propician incrementos de flujos inversos, aumentos de potencia reactiva, asimetrías en las señales de voltaje y corriente, disminución del factor de potencia, sobrecalentamiento en conductores y aislamientos, entre otros fenómenos. Los diagramas del modelo se vanalimentando con las restricciones técnicas del proceso de despacho tales como las de tipo térmico asociadas a la cargabilidad de algunos elementos como transformadores de potencia y restricciones de voltaje en líneas de transmisión.

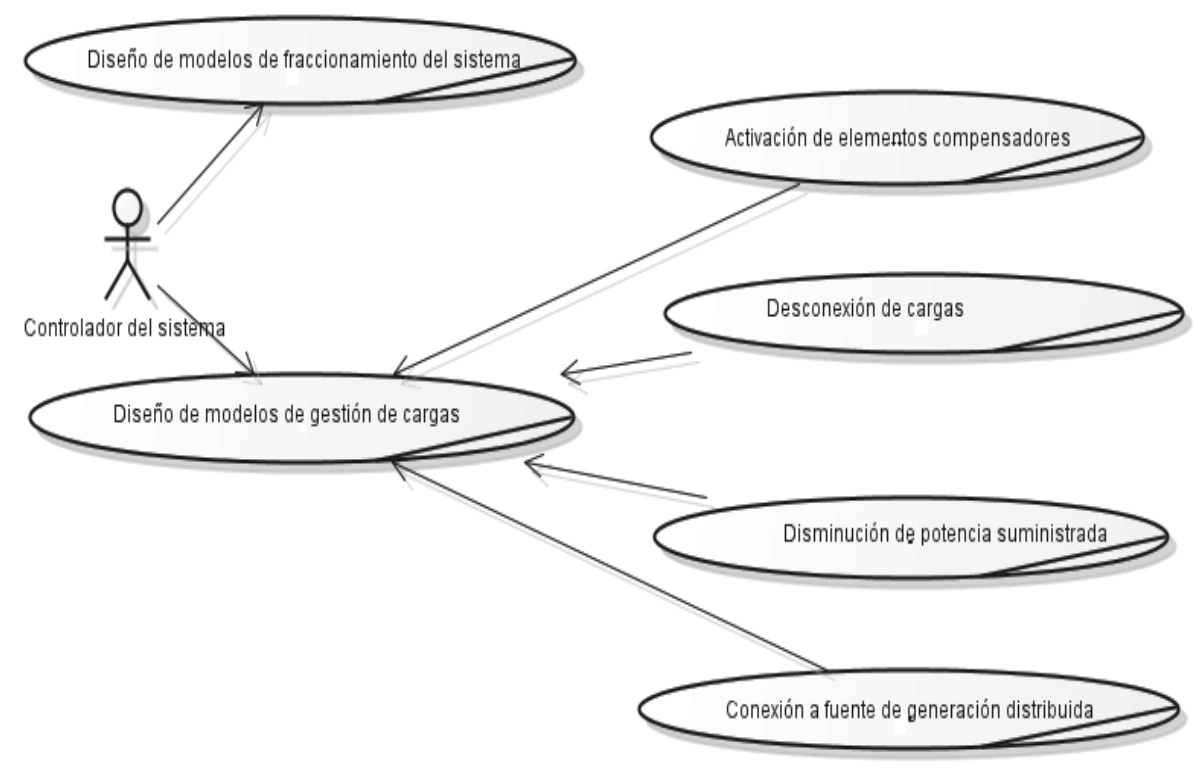

Figura 5. Diagrama de casos de Uso para el sistema de gestión de la red de distribución. Fuente: elaboración propia. 
En el diagrama de casos de Uso de la figura 5 se contemplan mecanismos de fraccionamiento o seccionamientode la red, que permiten aislar una falla que pueda llegar a afectar a toda la red. De la misma forma, se revisan otros mecanismos como la disminución de la potencia suministrada a las cargas abastecidas o deslastre de cargas; la desconexión de cargas no anunciadas en la red, generadoras de pérdidas no-técnicas; la activación o conexión formal de dispositivos correctores del factor de potencia, como bancos de condensadores y filtros activos para compensación de cargas no-lineales.

La fase de modelamiento se perfeccionará con la introducción final de criterios ligados al despacho con prelación o etiquetado para agentes de generación distribuida y cargas con alta responsabilidad en la red. El despacho preferente resulta de unas directrices claras de apoyo que el gobierno ofrece en la actualidad a las fuentes de energía renovables que lleguen a participar en un mercado común de energía junto a las centrales de generación convencional. La inclusión en el modelo de gestión del despacho energético de la opción de despacho preferente obliga a los investigadores al diseño de un proceso de adaptación dinámica del sistema de distribución de la zona.

En las etapas finales del desarrollo propuesto se elaboran los algoritmos del proceso de gestión con las variables de estado y las funciones-objetivo definidas previamente. A la elaboración de los algoritmos seguirá el diseño de la infraestructura de dispositivos de control y regulación que soportará la gestión automatizada del proceso de despacho en función de los requerimientos que se estipulen en cada momento particular de la distribución.

De acuerdo con la metodología de desarrollo propuesta a partir de UML, el desarrollo de las rutinas de comportamiento del sistema frente a fallos y en general situaciones de operación normal y anómala de la red de distribución se modela con los diagramas de clases y de secuencia para el sistema(figura 6).

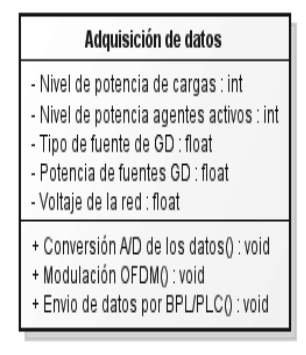

\begin{tabular}{|l|}
\hline \multicolumn{1}{|c|}{ Deslastre de carga en un nodo } \\
\hline - Potencia de cargas del nodo : int \\
- Nivel de prelación del nodo : int \\
- Nivel de prelación de las cargas : int \\
- Tipo de averia presentada : string \\
- Código de averia : int \\
\hline + Desconexión gradual de cargas 0 : void \\
+ Conexión de elementos de compensacióno : void \\
+ +Codificación de averia 0 : void \\
+ Evaluación de balance de potencia actual 0 : void \\
\hline
\end{tabular}

\begin{tabular}{|l|}
\hline \multicolumn{1}{|c|}{ Seccionamiento de la red de distribución } \\
\hline - Niveles de prelación de cargas : int \\
- Tipo de averia ocurrida : string \\
- Nivel de ponderación de la averia : int \\
- Número de secciones de la red : int \\
- Tipo de fraccionamiento : int \\
- Factor de potencia de cada sección : flloat \\
- Código de averia : int \\
\hline + Activación de seccionadores 0 : void \\
+Cálculo de balance de cargas 0 : void \\
+ Envio de infromación a base de datos 0 : void \\
+ Activación de compensadores de factor de potenciaO : void \\
\hline \hline \multicolumn{1}{c|}{ Conexión de fuentes de $G D$} \\
\hline
\end{tabular}

Figura 6. Diagrama de clases para el modelo de gestión de la red de distribución. Fuente: elaboración propia. 


\section{|investigación}

Para los ajustes finales de los diseños correspondientes se realizarán los protocolos de prueba de sistema mediante software de simulación para redes de potencia eléctrica. Estos protocolos deberán eva- luar la secuencia de acciones que se llevan a cabo durante los diferentes procedimientos que adopta el modelo de gestión,como se ilustra en el diagrama de secuencia de la figura 7.

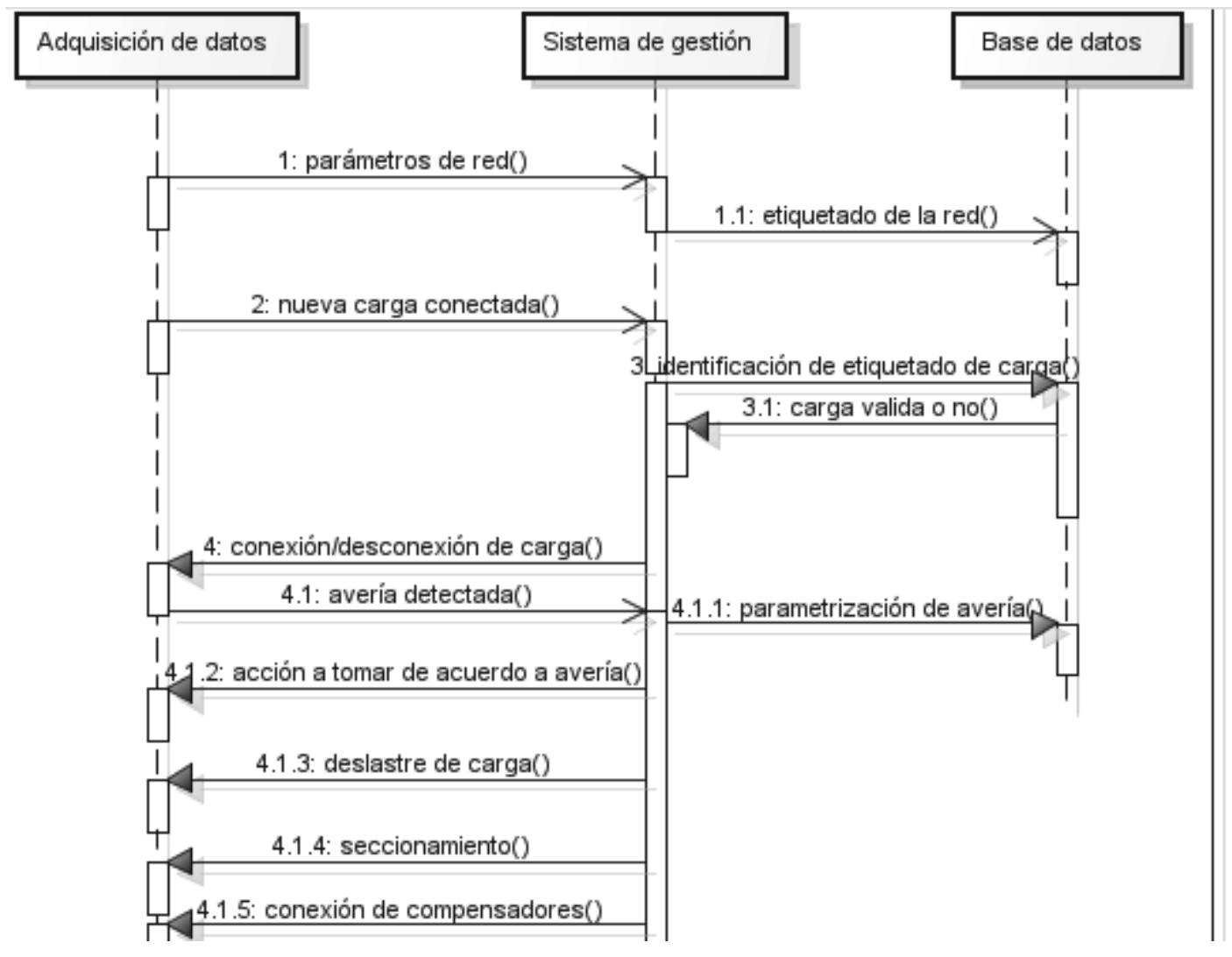

Figura 7. Diagrama de secuencia del sistema de gestión de la red de distribución.

Fuente: elaboración propia.

\section{PRUEBAS REALIZADAS DE LA TECNOLOGÍA BPL/PLC}

el escenario de pruebas escogido corresponde a un ambiente típico industrial donde conviven actuadores de diversos tipos, desde eléctricos y electroneumáticos hastadispositivos de red de datos convencionales como HUB, SWITCH y otros. Todo ello con el fin de obtener un medio con altos índices de ruido de amplio espectro y bajo condiciones de variabilidad en cuanto a parámetros eléctricos se refiere como factor de potencia, índices de distorsión armónica total en voltaje y corriente y las potencias reactivas y activas ligadas a los anteriores[20].Este escenario se resume en la figura 8. 


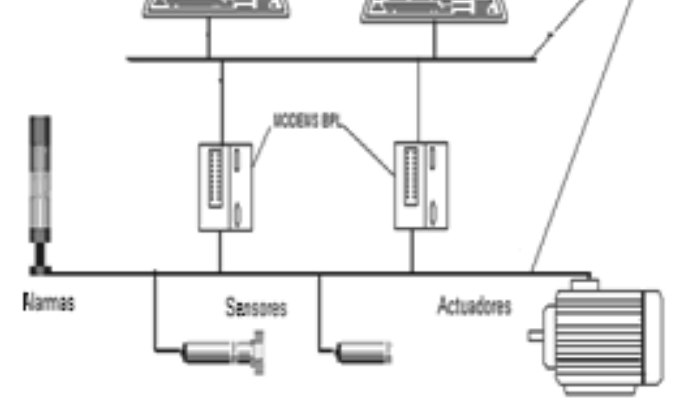

Figura 8. Escenario industrial de pruebas. Fuente: elaboración propia.

Debe señalarse que el ambiente industrial incluía un sistema de banda transportadora, equipada con sensores y actuadores neumáticos encargados de la colocación de piezas sobre ella. Esto, unido a la presencia de motores, permitía diseñar unescenario con ruido de amplio espectro sobre la red eléctrica.

El enlacede comunicación creado fue punto a punto entre los dos PC, uno portátil y otro de mesa. Ambos fueron conectados mediante el uso de los módems PLC a la red eléctrica, para enviar los datos a través de ella y dicho sea de paso, de allí mismo se abastecían de energía, constituyendo una carga adicional para el enlace y, como puede evidenciarse en los estudios previos realizados, una fuente considerable de armónicos en la red. La realización de estas pruebas se llevó a cabo con el fin de evaluar el comportamiento de la tecnología en el proceso de transmisión de los paquetes de información de extremo a extremo, tal y como se tendrá que realizar durante el proceso de control y gestión de la red de distribución. En trabajos previos del autor se observaron índices de pérdida de paquetes de $25 \%$ en momentos de establecimiento del enlace, pero que llegaron luego a niveles de 0 $\%$, al incluirse un número mayor de portadoras en la multiplexación OFDM (OrthogonalFrecuencyDivi-
sionMultiplexing) realizada [20].

\section{CONCLUSIONES Y RECOMENDACIONES}

El modelamiento del sistema de gestión de la red de distribución realizado permite plantear soluciones abiertas provistas de altos dinamismos, con interacción directa de los agentes activos generadores y las cargas consumidoras de la energía.

El lenguaje UML provee al sistema integral de la gestión de un carácter universal, abierto, sin ataduras a tecnologías de control específicas ni a enlaces de comunicación rígidos y poco convergentes.

La tecnología BPL/PLC ofrece un aporte importante a las nuevas infraestructuras de sistemas de gestión de la red de distribución con gran convergencia e integralidad,y con niveles de operatividad al mismo nivel de tecnologías de comunicación de espectro ensanchado de datos que utilizanInternet y redes de información como las industriales.

\section{REFERENCIAS}

[1] XM S.A. E.S.P, “Coordinación de operación del SIN (Sistema Interconectado Nacional)", conferencia auspiciada por XM S.A. Empresa de Servicios Públicos, marzo 11 de 2011.

[2] P. Palensky and D. Dietrich, "Demand side

management: Demand response, intelligent energy systems, and smart loads", ieee trans. ind. inform., vol. 7, no. 3, pp. 381388, aug. 2011.

[3] V. Calderaro, C. Hadjicostis, A. piccolo, and P. Siano, "Failure identification in smart grids based on Petri net modelin",

ieee trans. ind. electron., vol. 58, no. 10, pp. 4613-4623, oct. 2011.

[4] A. Yasser, "New Control Algorithms for the Distributed Generation Interface in GridConnected and Micro-grid Systems Uni- 
versity of Waterloo". Doctor of Philosophy in Electrical and Computer Engineering. Waterloo, Ontario, Canada, 2008.

[5] R. Lasseter, and P. Piagi. "Microgrids and Distributed Generation". Journal of Energy Engineering, American Society of Civil Engineers Microgrid, pp. 144-149, 2007.

[6] L. Yunwei, D.M. Vilathgamuwa and P. Chiang, "Design, analysis, and real-time testing of a controller for multibus microgrid system",power electronics, IEEE transactions on, vol.19, no.5, pp. 1195- 1204, 2004.

[7] A.M. Abdullah, "Calculation of Load Point Indices of Distribution Systems Due to Inserting a DG Device in Radial Systems", IEEE PES innovative smart grid technologies conference, 2012.

[8] SAE J2293: Estándar de requerimientos para vehículos eléctricos y sus fuentes de alimentación. [En linea], Disponible en:http://www.convertronic.net/Otros-productos/conectividad-y-comunicacionesentre-vehiculos-electricos-bevs-y-redeselectricas-inteligentes.

[9] J. Beristan, Inversores bidireccionales con aislamiento en alta frecuencia para aplicaciones de energías renovables, tesis doctoral, Universitat Politecnica de Catalunya, departamento de ingeniería electrónica, 2005.

[10] L. Degroote, B. Renders, B. Meersman, T. Vandoorm, and L. Vandevelde, "Power Quality Improvements through Power Electronic Interfaced Distributed Generation". SPEEDAM 2010 International Symposium on Power Electronics, Electrical Drives, Automation and Motion. IEEE Conference, 2010.

[11] R. Shaffer, "Power Electronic Harmonic

\section{investigación}

Analysis and Fundamentals of power electronics with MATLAB, power quality in electrical systems",first edition, Da vinci engineering series, Thomson learnin Inc, USA, 2007.

[12] D. Neacsu, "Power Switching Converters: Medium and High Power", Editorial CRC Press 2006-05-25, 2006 ISBN 10: 0824726251 .

[13] W. Yanjun and Y. Zhang, "Optimal algorithm of distribution network planning including distributed generation",electric utility deregulation and restructuring and power technologies, 2008. DRPT 2008. Third International Conference on, no. 4., pp.872-876,2008.

[14] S. Hun-Chul and K. Chul-Hwan, "An Adaptive Reclosing Algorithm Considering Distributed Generation", international journal of control, automation andsystems, vol. 6, no. 5, pp. 651-659, 2008.

[15] P. Lopez, P. Espeso, J. Medina and J. Drake, "Aplicaciones automatizadas en tiempo real basada en componentes software". [En línea]. Disponible en: http://www.ceautomatica.es/old/actividades/jornadas/ XXIV/documentos/tire/146.pdf 2004.

[16] C. Parra, R. Ruiz and P. Paz, "Modelado de procesos y desarrollo de sistemas software: Integración entre UML y EPC". [En línea]. Disponible en: http://www.researchgate. net/publication/45337484_Modelado_de_ procesos_y_desarrollo_de_sistemas_software_integracin_entre_UML_y_EPC, 2005.

[17] S. Perez, F. Orejas and N. Fuentes, "Automatización de la arquitectura de componentes genéricos usados en UML", 2005.
G. Engels, G., R. Heckel and S. Sauer, 


\section{investigación |}

“UML- A Universal Language?", ICATPN 2000, LNCS 1825, Berlin, Heidelberg, Springer-Verlag, 2000.

[19] E. Chacon, J. Velasco, y O. Rojas, "Principios de una metodología para integración empresarial bajo un enfoque holónico". [En línea]. Disponible en: http://www.unicauca. edu.co/ai/publicaciones/PMIEH.pdf, 2005.

[20] L. Arias, O. Flores and A. Chica, influence of harmonic in communication in LAN-BPL networks for different power factor, The $9^{\text {th }}$
Latin-American Congress on Electricity Generation and transmission - CLAGTEE, 2011. 